Research Article

\title{
Deep Learning-Based Computed Tomography Images for Quantitative Measurement of the Correlation between Epicardial Adipose Tissue Volume and Coronary Heart Disease
}

\author{
Han Wang $\mathbb{D}^{1}$, Hui Wang $\mathbb{D}^{2},{ }^{2}$ Zhonglve Huang $\mathbb{D}^{2},{ }^{2}$ Huajun Su $\mathbb{D}{ }^{2}$ Xiang Gao $\mathbb{D}^{2}, 2$ \\ and Feifei Huang $\mathbb{D}^{2}$ \\ ${ }^{1}$ Department of Cardiology, General Hospital of the Yangtze River Shipping, Wuhan, Hubei 430000, China \\ ${ }^{2}$ Department of Cardiology, Caidian District People's Hospital, Union Jiangbei Hospital, \\ Huazhong University of Science and Technology, Wuhan, Hubei 430100, China
}

Correspondence should be addressed to Feifei Huang; 2006010119@st.btbu.edu.cn

Received 12 May 2021; Revised 8 June 2021; Accepted 1 July 2021; Published 14 July 2021

Academic Editor: Gustavo Ramirez

Copyright (c) 2021 Han Wang et al. This is an open access article distributed under the Creative Commons Attribution License, which permits unrestricted use, distribution, and reproduction in any medium, provided the original work is properly cited.

The epicardial adipose tissue volume (EATV) was quantitatively measured by deep learning-based computed tomography (CT) images, and its correlation with coronary heart disease (CHD) was investigated in this study. 150 patients who underwent coronary artery CT examination in hospital were taken as research objects. Besides, patients from the observation group (group A) suffered from vascular stenosis, while patients from the control group (group B) had no vascular stenosis. The deep convolutional neural network model was applied to construct deep learning algorithm, and deep learning-based CT images were adopted to quantitatively measure EATV. The results showed that the sensitivity, specificity, accuracy, and area under the curve (AUC) of the deep learning algorithm were 0.8512, $0.9899,0.9623$, and 0.9813 , respectively. By comparison, the correlation results of the traditional George algorithm, Aslani algorithm, and Lahiri algorithm were all lower than those of the deep learning algorithm, and the difference was statistically substantial $(P<0.05)$. The epicardial adipose tissue volume of the observation group $(114.23 \pm 55.46)$ was higher markedly than the volume of the control group $(92.65 \pm 43.28)$, with a statistically huge difference $(P<0.05)$. The $r$ values of EATV with plaque properties and the number of stenosed coronary vessels were 0.232 and 0.268 in turn, both showing significant positive correlation. In conclusion, the sensitivity and other index values of deep learning algorithm were improved greatly compared with traditional algorithm. CT images based on deep learning algorithm achieved good blood vessel segmentation effects. In addition, EATV was closely related to the development of CHD.

\section{Introduction}

CHD is a heart disease in which coronary atherosclerosis causes stenosis or complete blockage of the lumen and the spasm of the coronary arteries, which in turn leads to myocardial ischemic necrosis. With the changes in people's diet and lifestyle, the incidence of CHD has risen sharply in recent years, which has seriously threatened people's health [1]. Therefore, the early prevention and treatment of CHD is a critical part of people's health management. This requires accurate and effective detection methods to cut off the health damage caused by CHD from the source based on the principle of "early detection and early treatment."
Existing studies have believed that the application of coronary artery percutaneous catheter digital subtraction radiography (DSA) for $\mathrm{CHD}$ has the best diagnostic effect, but DSA detection has many shortcomings, which not only causes wounds but also brings expensive testing costs. There are a lot of burdens, and these shortcomings greatly limit its wide application [2]. Multislice spiral CT coronary angiography is a new technology applied in DSA detection, which is noninvasive, simple to operate, high in image resolution, and effective in diagnosis. However, it is not suitable for clinical application due to the need to inject a larger dose of contrast agent during testing, which will adversely affect human kidney health. 
A new bio-friendly and efficient detection method has become the common expectation of doctors and patients. Medical imaging based on deep learning algorithm has been a hot topic of research by scholars in recent years. Many research results have been successfully applied to the differential diagnosis of clinical diseases, greatly enhancing the accuracy of doctors' diagnosis and treatment. In domestic studies, some scholars have applied deep learning algorithm in the segmentation of nerve cell images [3]. Some scholars have adopted deep learning algorithm in the differentiation of benign and malignant breast tumors [4]. In addition, some scholars have applied deep learning algorithm in pattern classification to achieve rapid classification of medical images [5]. In foreign studies, some scholars have applied deep convolutional neural network in the vascular segmentation of retina and achieved a good effect of vascular segmentation. Some scholars have applied three-dimensional convolutional neural network in the segmentation of brain pathological tissue, achieving a breakthrough in the study of brain tissue [6].

In order to obtain a good detection effect, an effective evaluation index is also needed. Studies have found that among the many pathogenic factors of cardiovascular disease, the volume of regional adipose tissue represented by the increase of visceral fat volume will increase, which is a significant risk factor. Yerramasu et al. [7] showed that the measurement of EATV by CT could be used as an index of the presence and severity of atherosclerosis in coronary arteries. EAT refers to the visceral fat deposited near the epicardium of the heart to the fibrous pericardium. It can secrete a variety of inflammatory factors and adipohormones and occupy $80 \%$ of the surface area of the heart. Besides, it is mainly distributed in the right ventricle, atrioventricular groove, and interventricular groove, and the rest are distributed around the free wall of the atrium and around the auricle. Regarding the formation mechanism of atherosclerosis, existing studies have speculated that the coronary arteries are connected to EATV by the adipocyte-vascular regulator. Therefore, it is more credible to use EATV as the evaluation index of CHD.

The correlation between EATV and CHD was explored in this study through CT images based on deep learning, so that to provide a more effective clinical detection method for the CHD diagnosis and treatment.

\section{Materials and Methods}

2.1. Vessel Segmentation Method Based on Deep Learning. Figure 1 shows the research direction of deep learning, while Figure 2 indicates the flowchart of convolutional neural network segmentation of blood vessels. The deep convolutional neural network model was employed to segment the cropped coronary vascular image blocks. One part clipped the original image, and the other part sampled the image before clipping and segmentation, so as to ensure that the local features have the global features for reference and obtain the precise segmentation of pixel points. The small blocks obtained by segmentation might be blood vessel images, and all small blocks were stitched together to get a complete coronary blood vessel segmentation image.
2.1.1. Preprocessing of Image Data. The coronary angiography images in this study were provided by hospital, with a total of 20. The original image was first processed by data amplification and then cropped according to medical image standards. After cropping, 18,720 images were obtained, of which 8,720 were used as the test set and 10,000 were used as the training set. Regarding the cropped image data, the ReLU function was applied to perform a unified normalization operation, so that it could be more easily recognized by the system. The normalization equation was as follows:

$$
x_{\text {normalization }}=\frac{x-\mu}{\max \{\delta, 1.0 / \sqrt{x}\}} \text {. }
$$

In equation (1), $\mu$ stood for the average of all pixels, $\sigma$ represented the standard deviation of the three-channel pixels, and the $x$-value was the total number of three-channel pixels.

2.1.2. Network Structure. In addition to the input layer, channel 1 had a five-layer structure, of which the convolutional layer was the first to the fourth layer, and the batch specification layer was the fifth layer. In the first, second, third, and fourth layers, the convolution kernel size was $5 * 5 * 32,5 * 5 * 64,3 * 3$, and $3 * 3$ in turn.

2.1.3. Parameter Optimization. In the training process of the network structure, the adaptive momentum optimization method was employed to achieve parameter optimization, which reduced the training time to the greatest extent and was more efficient than the stochastic gradient descent method. In addition, the Dropout technology was adopted to output unidentified neurons with a zero value to enhance robustness and reduce overfitting.

2.1.4. Loss Function. The objective function of the network was optimized by the cross-entropy loss function, and the function was defined as follows:

$$
\mathrm{CE}=-\sum_{X} p(x) \log (q(x)) .
$$

In equation (2), $p(x)$ and $q(x)$ stood for the true distribution of the sample, and the estimated probability obtained through training, respectively. When the crossentropy loss function was used for a binary classification task, its form was expressed as follows:

$$
\mathrm{CE}=-p(x) \log (q(x))-(1-p(x) \log ) .
$$

In equation (3), $p(x) \in\{ \pm 1\}$ specified the true class label of the sample and $q(x) \in[0,1]$ was the estimated probability of the model for the class with label $p(x)=1$.

2.1.5. Model Training. Thirty images were selected randomly, the effective circular area diameter of each image was 540 pixels, and the mask image was cut out and extracted based on the original image. Then, the DRIVE data set was adopted to train and test the model, which was used for retinal image 


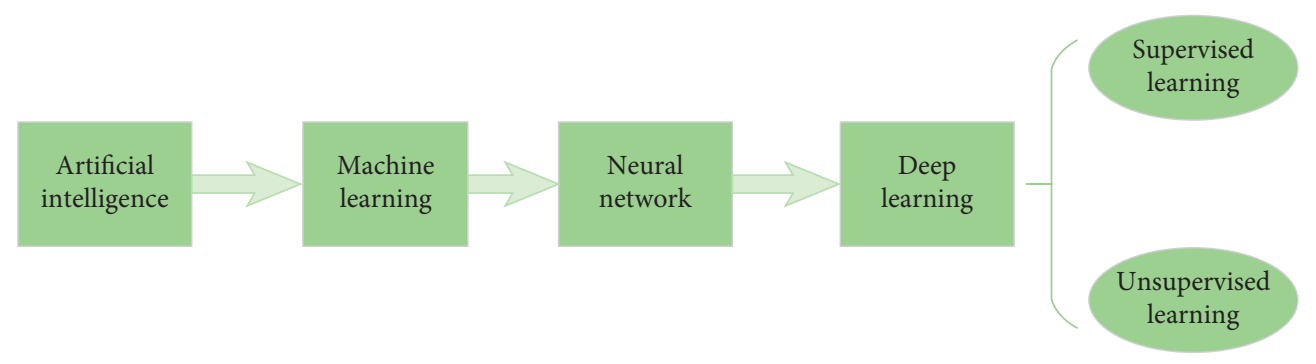

Figure 1: Research direction of deep learning.

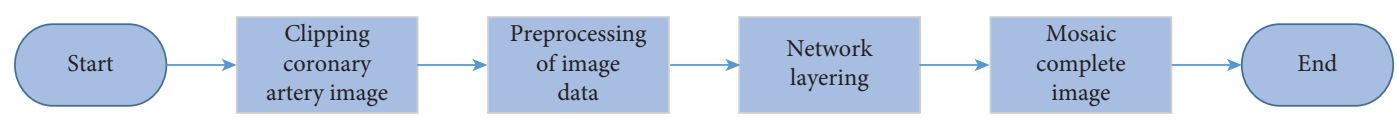

Figure 2: Flowchart of convolutional neural network segmentation of blood vessels.

segmentation, half of which was training set and the other half was test set. The training parameters were set as follows. Learning rate was 0.001 , batch size was 16 , iteration number was 10,000, and optimizer was Adam, Dropout 0.5.

2.2. Research Objects. From 2017 to 2019, 150 patients who underwent CT coronary angiography examinations in hospital were selected as the research objects. The hospital ethics committee had reviewed and authorized this study, and the research objects had understood this experiment and signed the informed consent forms.

The criteria for inclusion were defined to include patients who agreed to participate in this experiment, had the negative iodine allergy test results with no arrhythmia symptoms, had the scanned image quality that could be used to judge whether there was coronary artery stenosis, had no previous history of severe liver and kidney disease, cardiovascular and cerebrovascular disease, and diabetes, and had no missing data. Furthermore, the patients aged 22-74 years old, and more than $60 \%$ were male.

The criteria for exclusion were defined to include patients who underwent coronary stent implantation or bypass surgery due to cardiovascular disease, had the scanned image quality that could not be used to determine whether the coronary arteries were stenosis, had the past medical history of severe liver and kidney disease, cerebrovascular disease, and diabetes, and had the missing data.

2.3. Research Grouping. All the selected research objects were enrolled into the observation group (group A) and the control group (group B) based on the detection results of coronary CT angiography. The images of patients from group A showed stenosis of the main blood vessels, with a total of 83 cases. Besides, the images of group B presented no stenosis of the main blood vessels, and there were a total of 67 cases. Group A was further subdivided into two groups using different standards to conduct the following research. First, it was subdivided into a soft plaque group, a mixed plaque group, and a calcified plaque group based on the nature of plaque. The nature of plaque was defined by the plaque that caused the most severe coronary artery stenosis.
Second, it was subdivided into a single-vessel disease group, a double-vessel disease group, and a multivessel disease group according to the number of stenosed coronary vessel. What is more, the number of stenosed coronary vessels was defined by CT coronary angiography results.

\subsection{CT Scanning Method}

2.4.1. Preparation before Testing. All the tested patients should fast 4 hours before the test, and their basic information was measured and recorded such as the patients' weight before the test started. Besides, the patients were asked not to carry extra items that might cause image artifacts. They were informed in detail of all the examination procedures and possible reactions to relieve their unnecessary mental stress. In addition, it was necessary to perform multiple breathing exercises for the examinees until they could maintain a uniform and stable breathing evaluation rate, so as to avoid the interference caused by breathing artifacts on image quality to the maximum extent and prevent abnormal breathing fluctuations from affecting the image detection results. When the patient's resting heart rate exceeded 70 beats per minute, antiarrhythmic drugs (such as Betaloc) had to be taken 1 hour before the start of the test to make the heart rate normal and stable.

2.4.2. Scanning Method. A 64-slice spiral CT machine (SIEMENS definition 4.0) was employed to scan with the heart mode and gated method. The ioversol contrast agent was injected into the median cubital vein of each patient under high pressure at a flow rate of $5 \mathrm{~mL} / \mathrm{s}$. Moreover, the time density changes of the aortic root were detected through bolus tracking, and $110 \mathrm{HU}$ was used as the threshold to calculate the change of delay time during coronary artery scanning. Then, ioversol contrast agent was injected again into the cubital vein at the original rate with a dose of $1.0 \mathrm{~mL} / \mathrm{kg}$. The scanning range was from the trachea bifurcation to $10 \mathrm{~mm}$ below the septum. The scanning parameters included the following. The tube current was $300-800 \mathrm{~mA}$, tube voltage was $120 \mathrm{kV}$, the thickness of layer was $5 \mathrm{~mm}$, the layer thickness of reconstruction was $0.625 \mathrm{~mm}$, and DFOV was $12.0 \mathrm{~cm}$. 


\subsection{Image Analysis}

2.5.1. Coronary Artery Vascular Analysis. All scanned images were reconstructed using a deep learning-based blood vessel segmentation method, and the reconstructed images were transmitted to the syngo.via workstation. What is more, two radiologists reviewed the images in this study, who had extensive experience in reading the images. If there was a disagreement, the two would decide after consultation. Furthermore, the images of each patient were divided into 20 segments, and whether there were lesions, the nature of the plaque, and the tissue of the lesion should be evaluated and analyzed.

The coronary artery segmentation method was adopted to divide the coronary arteries by the American Heart Association. Besides, they were divided into first obtuse marginal branch; right coronary artery proximal, middle, distal, and posterior descending branch; the first and second diagonal branches; segment between the first and second obtuse marginal branches; the second large obtuse marginal branch; left main artery, left anterior descending branch, proximal, middle, and distal; the proximal of left circumflex branch; and the distal part of the main circumflex branch. When the vascular segment was less than $2 \mathrm{~mm}$, there was no reference value in coronary artery assessment, so no in-depth study was needed. According to the classification standards established by the American Heart Association, atherosclerotic plaques were divided into three types, namely, calcified plaque, mixed plaque, and noncalcified plaque. What is more, the criterion for defining calcified plaque was CT value $>130 \mathrm{HU}$. When the calcified area of plaque was less than half, it was mixed plaque. When low-density plaque appeared in multiple arterial stenosis images, it was soft plaque, also known as noncalcified plaque. In addition, the arteries were often divided into four categories according to the number of stenosed coronary vessel in the results of CT coronary angiography, including multiple-vessel disease, double-vessel disease, single-vessel disease, and no disease.

2.5.2. Measurement of Epicardial Adipose Tissue Volume. The built-in software of the SIEMENS definition 4.0 multislice spiral CT machine was applied to quantitatively measure the volume of each research object's EAT. The measuring physician manually outlined the surrounding boundary at an interval of $6 \mathrm{~mm}$ in the area where the heart was located and obtained fat images in the window width range of $-250-30 \mathrm{Hu}$. Then, the measuring tool was employed to accurately measure the fat volume, and milliliters $(\mathrm{mL})$ was used as the measurement volume unit. The selected measurement range was the largest to the pulmonary artery bifurcation, the smallest to the septal muscle, the anterior part to the sternum, and the posterior part to the descending aorta and esophagus. When the adipose tissue volume measured by the measuring tool was within the set CT threshold value range, it was recorded as EATV. On this basis, the total volume within the currently checked range was measured and recorded. In addition, the ratio of EATV to total volume was calculated, which should be recorded and analyzed.
2.6. Statistical Analysis. The data obtained in this study were input into Excel 2010 software and the corresponding database was established. The statistical analysis of this study adopted SPSS 13.0 statistical software, and measurement data were represented as mean \pm standard deviation $(\bar{x} \pm s)$. When conforming to normal distribution, comparison between two groups was detected by independent sample $t$-test, and the comparison among multiple groups was tested by one-way analysis of variance (ANOVA). Besides, the skewed distribution was represented by $M(\mathrm{Q})$, pair comparison was detected by Mann-Whitney $U$ test, and Kruskal-Wallis test was used for comparison among multiple groups. Spearman's correlation was adopted to analyze the correlation of EATV with plaque properties and the degree of coronary stenosis. The area under the receiver operating characteristic curve (ROC) (Az) was applied to analyze the correlation between EATV and CHD. The count data was represented by rate (\%) and analyzed by $X^{2}$ test. All data were considered statistically substantial with $P<0.05$, while $P>0.05$ indicated that the difference was not statistically remarkable.

\section{Results}

3.1. Algorithm Index Analysis. In the DRIVE data, the deep learning algorithm and the traditional algorithm were compared and analyzed, and the indexes included sensitivity, specificity, accuracy, and area under the curve (AUC). The results are shown in Table 1. The sensitivity, specificity, accuracy, and AUC of the deep learning algorithm were $0.8512,0.9899,0.9623$, and 0.9813 , respectively. By comparison, the correlation results of the traditional George algorithm, Aslani algorithm, and Lahiri algorithm all reduced hugely in contrast to those of the deep learning algorithm $(P<0.05)$.

Figure 3 indicates that the blood vessel segmentation method based on deep learning could accurately segment capillaries that could not be distinguished, and the segmentation effect was good. Figures 3(a) and 3(b) show the vascular segmentation images of the same coronary heart disease (CHD) patient, who was a 47-year-old male with a weight of $62 \mathrm{~kg}$ and a height of $178 \mathrm{~cm}$.

\subsection{Comparison on General Information of All Patients.} Figure 4 presents the comparison results of general information of patients from both groups. The mean age $(58.24 \pm 7.36$ years old) and the male proportion $(71.1 \%)$ in patients from the observation group were higher than those of the control group (51.28 \pm 7.78 years old and $64.2 \%)$, and the difference between the two groups was statistically marked $(P<0.05)$. The body weight of the observation group $(72.16 \pm 9.84 \mathrm{~kg})$ was higher than the weight of the control group $(71.33 \pm 10.57 \mathrm{~kg})$, but there was no great difference between the two groups $(P>0.05)$.

3.3. Epicardial Adipose Tissue Volume Results of Patients from the Two Groups. Figure 5 shows the typical epicardial fat volume CT images of the observation group and the control 
TABLE 1: Index analysis on deep learning algorithm and traditional algorithm.

\begin{tabular}{|c|c|c|c|c|}
\hline Method & Sensitivity & Specificity & Accuracy & AUC \\
\hline Azzopardi et al. [8] & 0.7612 & 0.9623 & 0.9426 & 0.9588 \\
\hline Aslani and Sarnel [9] & 0.7588 & 0.9783 & 0.9548 & 0.9613 \\
\hline Lahiri et al. [10] & 0.7534 & 0.9724 & 0.9465 & 0.9529 \\
\hline Deep learning algorithm & 0.8512 & 0.9899 & 0.9623 & 0.9813 \\
\hline
\end{tabular}

Note. AUC meant the area under the ROC curve. Accuracy $=$ true positive $/($ true positive + false positive); sensitivity $=$ true positive $/($ true positive + false negative); and specificity $=$ true negative $/($ false positive + true negative $)$.

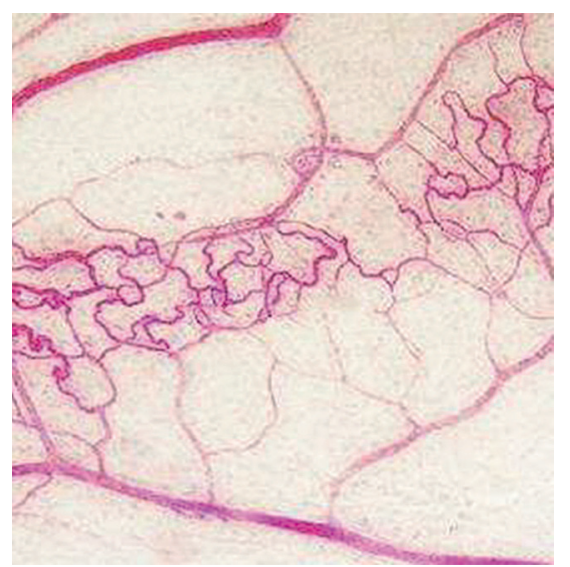

(a)

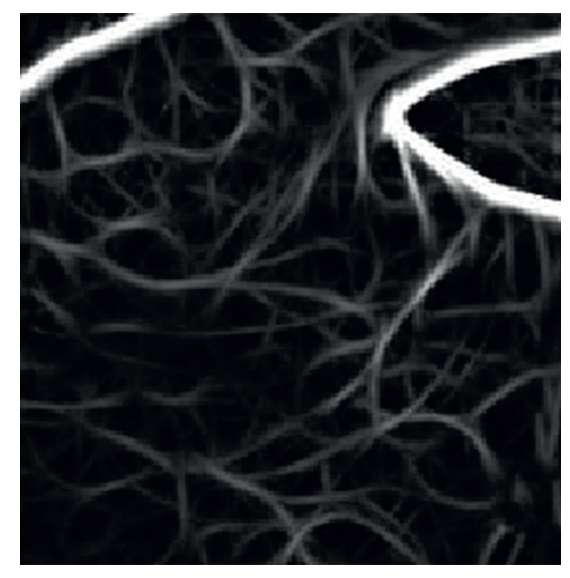

(b)

FIgURE 3: Small blood vessels in coronary arteries. (a) The original image and (b) the algorithm segmented image.

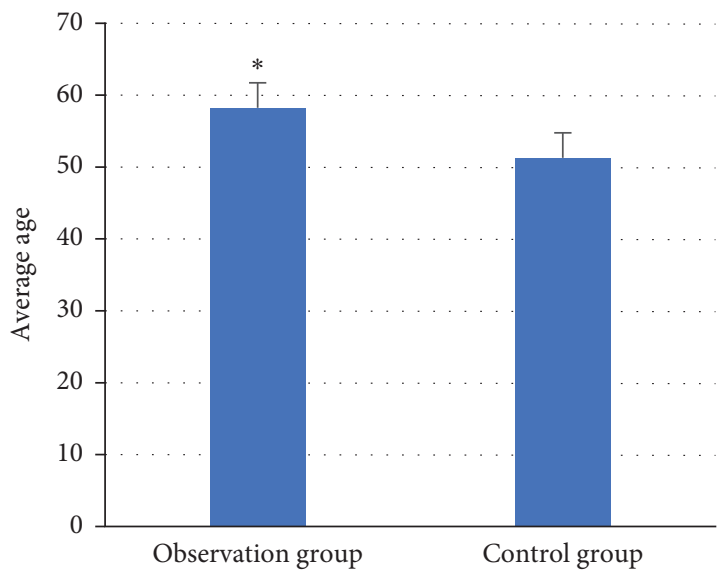

(a)

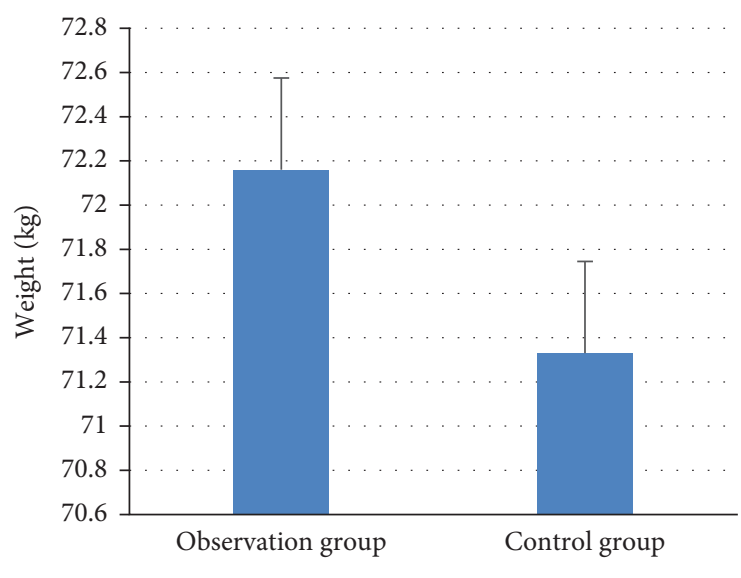

(b)

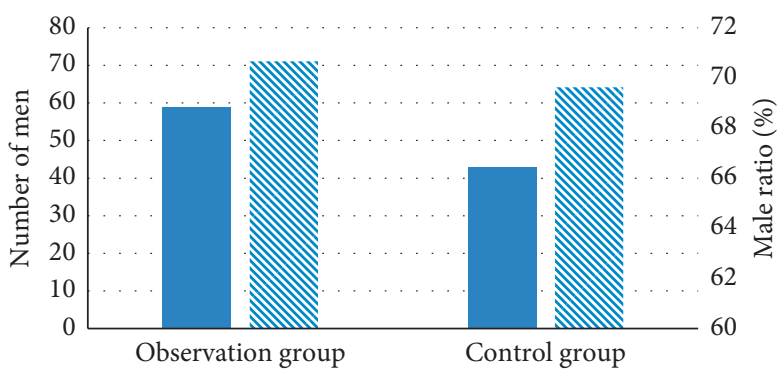

Number of men

s Male ratio

(c)

Figure 4: Comparison on general information of patients from both groups. (a) The comparison results of average age, (b) the comparison results of weight, and (c) the comparison results of the number and proportion of men. ${ }^{*}$ The difference was statistically marked compared with group B $(P<0.05)$. 


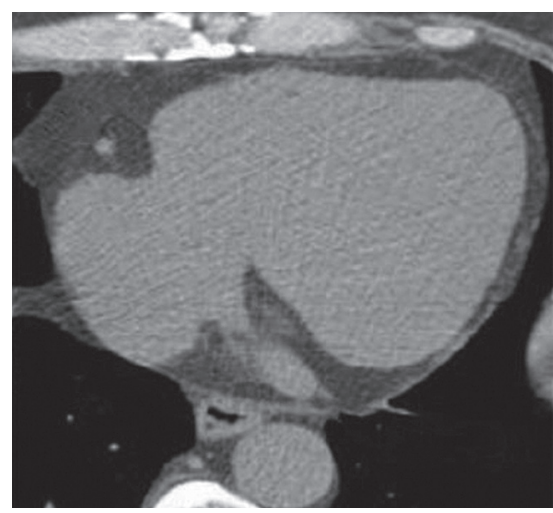

(a)

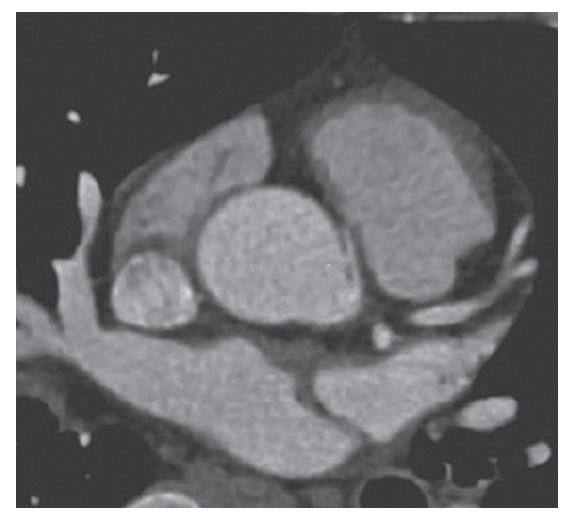

(b)

FIgUre 5: CT images of EATV. (a) The control group and (b) the observation group.

group. Through comparison, it could be clearly observed that the area of high-density shadow of the epicardial fat in the CT images of the observation group was larger than that of the control group. The EATV levels between the observation group and the control group were compared and analyzed, and the results are presented in Figure 6. The values of EATV and TV in patients from the observation group were $114.23 \pm 55.46 \mathrm{~mL}$ and $911.35 \pm 227.78 \mathrm{~mL}$ in sequence, which were higher obviously than the control group $(92.65 \pm 43.28 \mathrm{~mL}$ and $833.87 \pm 162.66 \mathrm{~mL})(P<0.05)$. After calculation, the EATV/TV values of the observation group and the control group were $0.12 \pm 0.05$ and $0.11 \pm 0.06$, so there was no statistically obvious difference between the two groups $(P>0.05)$. It indicated that the value of EATV would increase when patients suffered from coronary artery stenosis.

3.4. Correlation between Epicardial Adipose Tissue Volume and Coronary Stenosis. There was a positive correlation of EATV and the degree of stenosis $(r=0.317$ and $P=0.004)$, which could be obtained from Table 2 .

3.5. Relationship of Epicardial Adipose Tissue Volume with the Nature of Plaque and the Number of Stenosed Coronary Vessels in Patients from Group A. The EATV of patients from group A was not affected by the nature of coronary plaque and the number of stenosed coronary vessels and was always greater than that of group $\mathrm{B}(P<0.05)$. Among the groups of different plaque properties, the highest EATV of mixed plaque was $118.58 \pm 54.35 \mathrm{~mL}$, and the difference between the groups was statistically significant $(P<0.05)$, as shown in Figure 7. Among the groups of different lesions, the highest EATV in the double-vessel lesion group was $121.35 \pm$ $58.58 \mathrm{~mL}$, and the difference between the groups was statistically substantial $(P<0.05)$, as shown in Figure 8 .

3.6. Spearman's Correlation of Epicardial Adipose Tissue Volume with the Nature of Plaque and the Number of Stenosed Coronary Vessels. The $r$ values of EATV with the nature of plaque and the number of lesions were 0.232 and 0.268 , respectively, showing a significant positive correlation. Therefore, it could be adopted to predict coronary atherosclerosis (Table 3 ).

3.7. ROC Curve Analysis of the Authenticity of Coronary Artery Judged by Epicardial Adipose Tissue Volume. The results of coronary CT angiography were taken as the detection standard, and when EATV was used to determine the coronary artery stenosis in the 67 cases of group B and 83 cases of group A, a series of values related to sensitivity and specificity were generated within the defined range of different critical values. ROC curves were drawn with $1-$ specificity as the abscissa axis and sensitivity as the vertical axis. Judging by the Youden index (Youden's index = sensitivity + specificity -1 ), the cut-off value of coronary artery stenosis EATV was $95.32 \mathrm{~mL}$, the ROC curve area in the figure was 0.645 , the $95 \%$ confidence interval was $0.517-0.726$, and the $P$ value was 0.002 (Figure 9).

\section{Discussion}

In the noninvasive examination of $\mathrm{CHD}, \mathrm{CT}$ imaging quantitatively measures the volume of epicardial fat to diagnose CHD. The detection effect is good, and it is necessary to further promote the application. Many scholars have shown that the development of coronary atherosclerosis is related to the excessive increase of epicardial fat volume and adipokines [11]. In this study, the EATV, total cardiac measurement volume, EATV/total measurement volume, and CT value of EATV in groups A and B were compared, showing that the EATV of group A was higher markedly than the volume of group B $(P<0.05)$. Miyoshi et al. [12] found that the increase in EATV was associated with the composition of coronary artery injury plaque and was not affected by abdominal visceral fat tissue (VAT), coronary artery calcification (CAC) score, and body mass index (BMI). Motoyama et al. [13] disclosed that the degree of damage to the coronary plaque injury plate was closely related to EATV, and the incidence of acute coronary syndrome in patients with CHD could be predicted based on EATV to realize the prevention and treatment of the disease. 


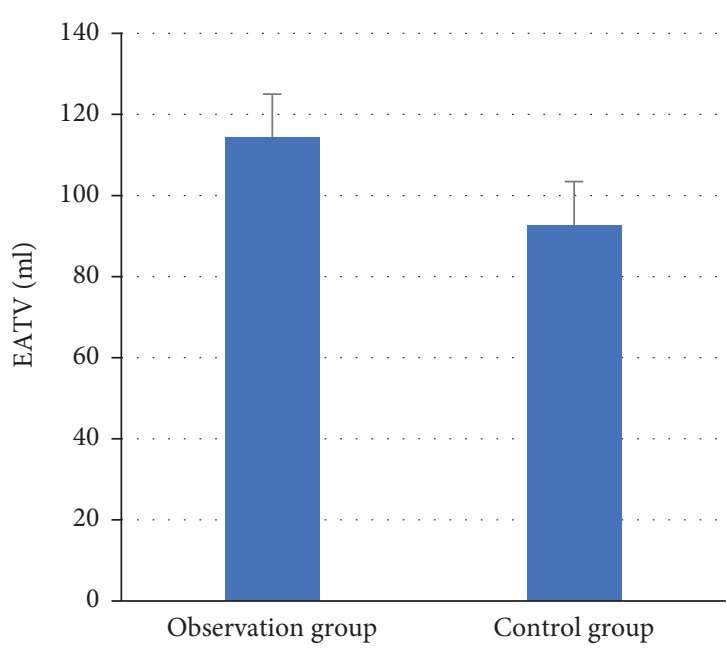

(a)

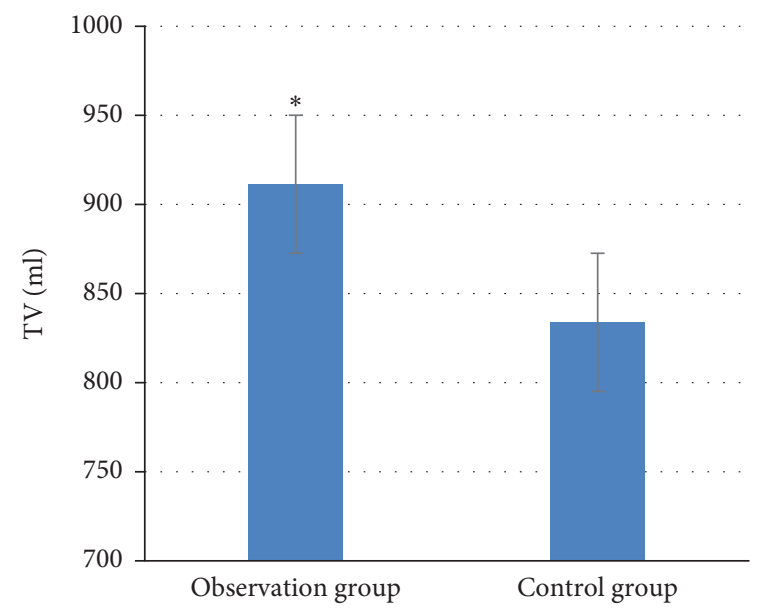

(c)

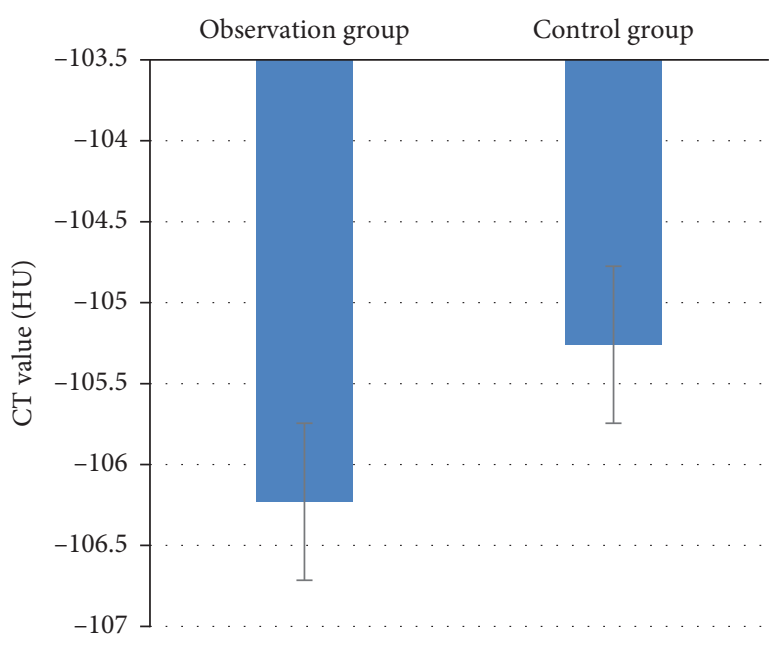

(b)

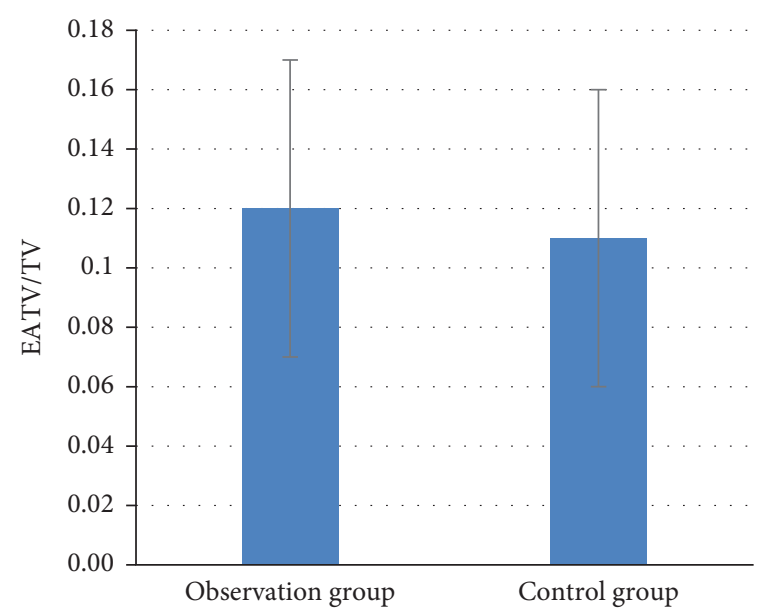

(d)

FIgUre 6: Comparison on the EATV results of patients from group A and B. CT value: CT value of EATV; TV: total volume of the delineated heart; EATV/TV: ratio of the epicardial adipose tissue volume to the total volume of delineated heart. ${ }^{*}$ The difference was statistically substantial by comparing with group B $(P<0.05)$.

TABLE 2: Spearman's correlation analysis between EATV level and the degree of coronary artery stenosis in patients from group A.

\begin{tabular}{lcc}
\hline Index & $r$ value & $P$ value \\
\hline EATV $(\mathrm{mL})$ & 0.317 & 0.004 \\
\hline
\end{tabular}

Group A was divided into groups in this study according to the nature of plaque and the number of stenosed coronary vessels. It was found that there were obvious differences in the value of EATV in each group. Then, Spearman's correlation was used to analyze the correlation between EATV and CHD. It indicated that there was a positive relation of EATV with the number of stenosed coronary vessels and the nature of plaque. In addition, the results also revealed that there was no statistical difference between groups A and B in the epicardial adipose CT value, EATV/measured total volume value, suggesting that EATV was an independent risk factor for CHD. Thus, it could be used as a clinical detection index for CHD, which was basically consistent with the research findings of [14]. In the assessment of the

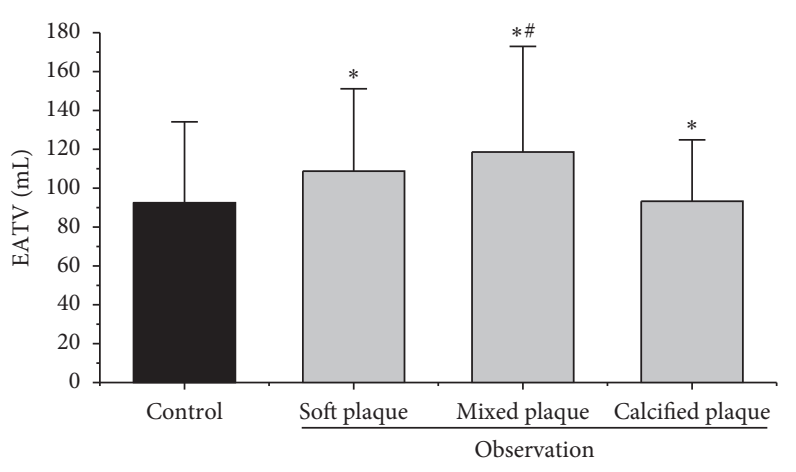

FIgURE 7: Comparison on EATV with different plaque properties in patients from the observation group. ${ }^{*} P<0.05$ compared with group $B ;{ }^{\#} P<0.05$ in contrast to soft plaque and calcified plaque in patients from group A.

patient's future coronary artery health, it is feasible to use high EATV as a risk factor, and it can increase the clinical predictive value of another detection index-calcification 


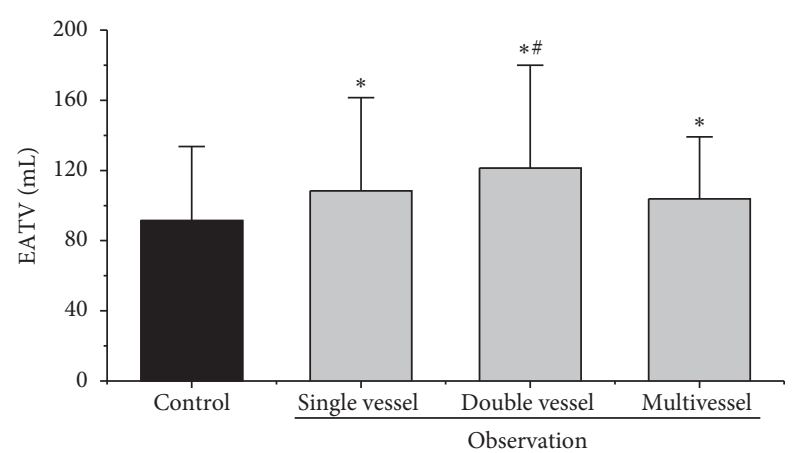

Figure 8: Comparison on the EATV of different stenosed coronary vessel in group A. ${ }^{*} P<0.05$ compared to group $\mathrm{B} ;{ }^{\#} P<0.05$ in contrast to single-vessel disease and multiple-vessel disease.

TABLE 3: Spearman's correlation of EATV with the nature of plaque and the number of stenosed coronary vessel.

\begin{tabular}{lcc}
\hline Index & $r$ value & $P$ value \\
\hline Nature of plaque & 0.232 & 0.004 \\
Stenosed coronary vessel & 0.268 & 0.001 \\
\hline
\end{tabular}

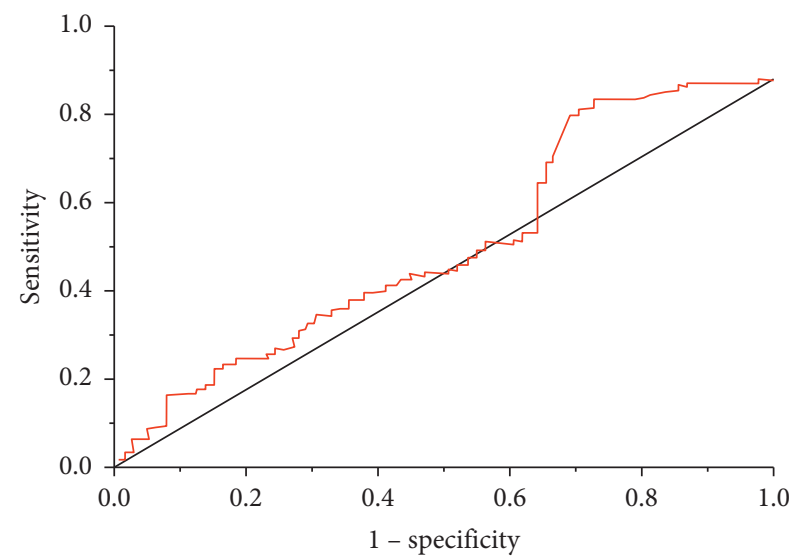

— Epicardial adipose tissue volume

— Reference line

FIgURe 9: ROC curve of epicardial adipose tissue volume.

score for CHD [15]. In recent years, bio-friendly imaging technologies such as CT have played an increasingly critical role in the clinical detection of cardiovascular diseases through the quantitative measurement of EATV [16].

\section{Conclusion}

In this study, CT images based on deep convolutional neural network learning algorithms were applied to detect EATV, and the correlation between EATV and CHD was explored. The results disclosed that the sensitivity and other index values of the deep learning algorithm were improved significantly compared with the traditional algorithm. The volume of epicardial fat was closely related to the development of $\mathrm{CHD}$, and it can be used as a marker for $\mathrm{CHD}$ detection. However, this research still has shortcomings, such as too single research indexes, small research scope, insufficient sample size, and insufficient representativeness of the research. Therefore, in future research, the diversity of research indicators and the extensiveness of research samples will be strengthened to make the research results more representative. It is hoped that a more effective detection method can be provided for the clinical diagnosis and treatment of $\mathrm{CHD}$ in the future.

\section{Data Availability}

No data were used to support this study.

\section{Conflicts of Interest}

The authors declare that they have no conflicts of interest regarding the publication of this paper.

\section{Authors' Contributions}

Han Wang and Hui Wang contributed equally to this work.

\section{References}

[1] O. Ghekiere, R. Salgado, and N Buls, "Image quality in coronary CT angiography: challenges and technical solutions," British Journal of Radiology, vol. 90, no. 1072, Article ID 20160567, 2017.

[2] H. Mírka, J. Ferda, and J. Baxa, "Assessment of myocardial enhancement during coronary CT angiography in critically ill patients," European Journal of Radiology, vol. 85, no. 10, pp. 1909-1913, 2016.

[3] T. Mochizuki, S. Hosoi, H. Higashino, Y. Koyama, T. Mima, and K. Murase, "Assessment of coronary artery and cardiac function using multidetector CT, "Seminars in Ultrasound, CT and MRI, vol. 25, no. 2, pp. 99-112, 2004.

[4] P. Schoenhagen, S. S. Halliburton, A. E. Stillman et al., "Noninvasive imaging of coronary arteries: current and future role of multi-detector row CT," Radiology, vol. 232, no. 1, pp. 7-17, 2004.

[5] D. Andreini, G. Pontone, S. Mushtaq et al., "Image quality and radiation dose of coronary CT angiography performed with whole-heart coverage CT scanner with intra-cycle motion correction algorithm in patients with atrial fibrillation," $E u$ ropean Radiology, vol. 28, no. 4, pp. 1383-1392, 2018.

[6] X. Xie, M. J. Greuter, J. M. Groen et al., "Can nontriggered thoracic CT be used for coronary artery calcium scoring? a phantom study," Medical Physics, vol. 40, no. 8, Article ID 081915, 2013.

[7] A. Yerramasu, S. Venuraju, S. Atwal, D. Goodman, D. Lipkin, and A. Lahiri, "Radiation dose of CT coronary angiography in clinical practice: objective evaluation of strategies for dose optimization," European Journal of Radiology, vol. 81, no. 7, pp. 1555-1561, 2012.

[8] G. Azzopardi, N. Strisciuglio, M. Vento, and N. Petkov, "Trainable COSFIRE filters for vessel delineation with application to retinal images," Medical Image Analysis, vol. 19, no. 1, pp. 46-57, 2015.

[9] S. Aslani and H. Sarnel, "A new supervised retinal vessel segmentation method based on robust hybrid features," Biomedical Signal Processing and Control, vol. 30, no. 9, pp. 1-12, 2016. 
[10] A. Lahiri, A. G. Roy, D. Sheet, and P. K. Biswas, "Deep neural ensemble for retinal vessel segmentation in fundus images towards achieving label-free angiography," in Proceedings of the Annual International Conference of the IEEE Engineering in Medicine and Biology Society, pp. 1340-1343, IEEE Engineering in Medicine and Biology Society, Orlando, FL, USA, August 2016.

[11] A. Sabarudin, Z. Sun, and K.-H. Ng, "Radiation dose associated with coronary CT angiography and invasive coronary angiography: an experimental study of the effect of dosesaving strategies," Radiation Protection Dosimetry, vol. 150, no. 2, pp. 180-187, 2012.

[12] T. Miyoshi, K. Ejiri, K. Kohno et al., "Effect of remote ischemia or nicorandil on myocardial injury following percutaneous coronary intervention in patients with stable coronary artery disease: a randomized controlled trial," International Journal of Cardiology, vol. 236, pp. 36-42, 2017.

[13] S. Motoyama, T. Kondo, H. Anno et al., "Atherosclerotic plaque characterization by $0.5-\mathrm{mm}$-slice multislice computed tomographic imaging comparison with intravascular ultrasound," Circulation Journal, vol. 71, no. 3, pp. 363-366, 2007.

[14] A. Yerramasu, D. Dey, S. Venuraju et al., "Increased volume of epicardial fat is an independent risk factor for accelerated progression of sub-clinical coronary atherosclerosis," Atherosclerosis, vol. 220, no. 1, pp. 223-230, 2012.

[15] W. Dong, J. Li, H. Mi, X. Song, J. Jiao, and Q. Li, “Relationship between collateral circulation and myocardial viability of 18FFDG PET/CT subtended by chronic total occluded coronary arteries," Annals of Nuclear Medicine, vol. 32, no. 3, pp. 197-205, 2018.

[16] H. N. Lee, J. I. Kim, and J.-M. Cho, "Bilateral coronary artery to left ventricular fistula: CT demonstration of drainage via a single, common channel," Cardiology in the Young, vol. 27, no. 4, pp. 782-783, 2017. 